\title{
HWA MEASUREMENT OF TURBULENT DIFFUSION OF A SCALAR QUANTITY
}

\begin{abstract}
Pavel ANTOŠ•
Abstract: The paper deals with simultaneous measurement of the velocity and the scalar quantity by means of hot-wire anemometry. Statistical moments of the scalar quantity fluctuations can be obtained employing a dual hot-wire probe. An evaluation procedure of the quantity mean values and fluctuations is described. Results from the molar concentration measurement in binary-gas mixture are shown.
\end{abstract}

\section{INTRODUCTION}

Hot-wire anemometry is suitable method for simultaneous measurement of the velocity and the scalar quantity of the flow. A probe with two sensors is employed in such measurements. Statistical moments of the scalar quantity, e.g. the fluid temperature $T_{a}$ or the molar concentration $C$ of binary-gas mixture, can be obtained.

\section{THEORETICAL BACKGROUND}

A heated element is, in principle, sensitive not only to the velocity $u$ but also to the thermophysical properties of the flow. Anemometer output voltage $E=E\left(u, T_{w}, T_{a}, C, \lambda, \rho, \mu, I_{w}, d_{w}, \ldots\right)$ is dependent on many variables. Introducing general variable $\psi$ as an observed scalar quantity we can write a cooling law of the sensor in the forms:

$$
\begin{gathered}
N u=N u(R e, \psi) \\
E=E(u, \psi)
\end{gathered}
$$

A dual hot-wire probe consists of two sensors (subscript $j=1,2$ ). We assume the same velocity and scalar quantity at measurement "point" - on both sensors.

$$
u=u_{1}=u_{2}, \quad \psi=\psi_{1}=\psi_{2} .
$$

Relation (1) in dimensional form written for both sensors creates a system of two equations:

$$
E_{j}=f_{j}(u) g_{j}(\psi) .
$$

Non-linear functions $f$ and $g$ depends on used type of cooling law; parameters result from calibration of the sensors. Solving the system numerically we obtain instantaneous values of observed quantities.

To reduce computational demands there is useful to separate evaluation of mean values and fluctuations. At each probe location we compute mean values $\bar{u}, \bar{\psi}$ from the mean voltage

- Ing. Pavel Antoš, Ph.D., Institute of Thermomechanics AS CR, v.v.i., Dolejškova 5, 18200 Praha 8. antos@it.cas.cz 
Then we compute fluctuations by following relations. Sensitivities to the velocity $u$ and the scalar quantity $\psi$ can be computed from the calibration:

$$
S_{u j}=\left.\frac{\partial E_{j}}{\partial u}\right|_{\bar{\psi}, \bar{u}} \quad, \quad S_{\psi j}=\left.\frac{\partial E_{j}}{\partial \psi}\right|_{\bar{\psi}, \bar{u}} .
$$

Anemometer output voltage $E$ can be written as follows:

$$
E_{j}(u, \psi)=\bar{E}_{j}+S_{u j} d u+S_{\psi j} d \psi \text {. }
$$

A system of two equations (5) written for both sensors leads into:

$$
u=\bar{u}+\frac{S_{\psi 2} d E_{1}-S_{\psi 1} d E_{2}}{S_{u 1} S_{\psi 2}-S_{u 2} S_{\psi 1}} \quad, \quad \psi=\bar{\psi}+\frac{S_{u 1} d E_{2}-S_{u 2} d E_{1}}{S_{u 1} S_{\psi 2}-S_{u 2} S_{\psi 1}} .
$$

The system is badly conditioned if $\left(S_{u 1} S_{\psi 2}-S_{u 2} S_{\psi 1}\right) \rightarrow 0$, what can be seen from eq. (6). Correct setup of simultaneous measurement therefore requires different ratios of sensitivities for both sensors:

$$
\frac{S_{u 1}}{S_{\psi 1}} \neq \frac{S_{u 2}}{S_{\psi 2}}
$$

This can be achieved by wise choice of experimental setup. Sensitivities to the observed quantities depend on the geometry and temperature of the sensor $\left(d_{w}, I_{w}, T_{w}\right)$. One should also pay attention to orientation of sensors and directional characteristics of the probe because of requirement of equality of effective velocities for both sensors.

\section{Concentration Measurement}

Procedure described above was applied on simultaneous measurement of the velocity and the molar concentration in binary-gas mixture. The mean values and the variances of the concentration were evaluated. A case of polynomial shaped 2D hill with the line source of tracer gas was studied. Shape of the hill was taken from Almeida at al. [5]. Cross section of the channel was of a width of $0.1 \mathrm{~m}$ and a height of $0.25 \mathrm{~m}$. The velocity of the main flow was set at $6 \mathrm{~m} . \mathrm{s}^{-1}$. An admixture gas $\mathrm{CO}_{2}$ as a tracer enters the main airflow upstream the hill at $x=-140 \mathrm{~mm}$. Volume rate of admixture was set at $5 \mathrm{e}-4 \mathrm{~m}^{3} \cdot \mathrm{s}^{-1}$ that corresponds with velocity in the slot of $5 \mathrm{~m} . \mathrm{s}^{-1}$. The results are presented in the positions of $x=(0 ; 10 ; 20 ; 30 ; 40 ; 50 ; 70 ; 90 ; 110 ; 130 ; 150) \mathrm{mm}$ (see Fig. 1$)$.

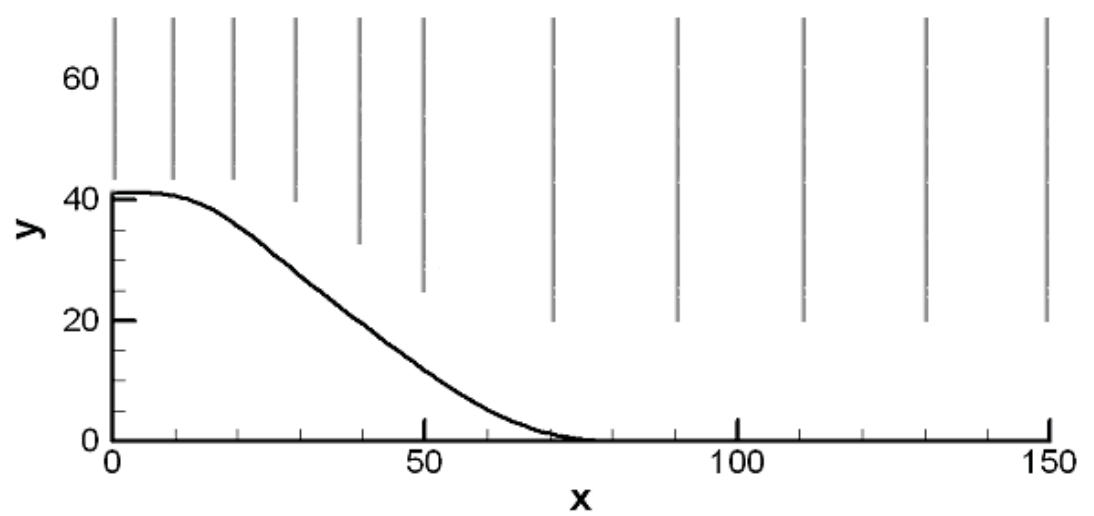

Figure: 1. Vertical traversing ( $x$-positions).

A three-sensor HWA probe was used. Only two wires were employed for this measurement (parallel sensors, distance between wires is approximately $0.5 \mathrm{~mm}$; W1 has a Pt-Rh wire of the diameter $d_{1}=10 \mu \mathrm{m}$ and the length $l_{1}=1.22 \mathrm{~mm}$; W2 has a 
tungsten wire of the diameter $d_{2}=2.5 \mu \mathrm{m}$ and the length $I_{2}=1.54 \mathrm{~mm}$; operating wire temperatures are $T_{w 1}=773 \mathrm{~K}, T_{w 2}=473 \mathrm{~K}$ ). Calibration in mixture air- $\mathrm{CO}_{2}$ at several values of concentration $C=0-0.91$ and several flow velocities $u=3-20 \mathrm{~m} \cdot \mathrm{s}^{-1}$ was performed in a hermetic close-circuit rig.

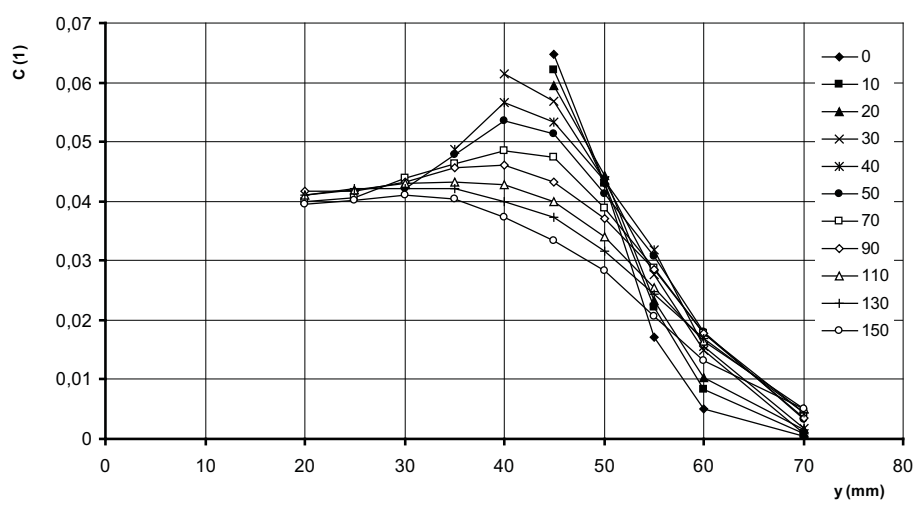

Graph 2: Mean value of the concentration $\bar{C}$ ( $x$-profiles).

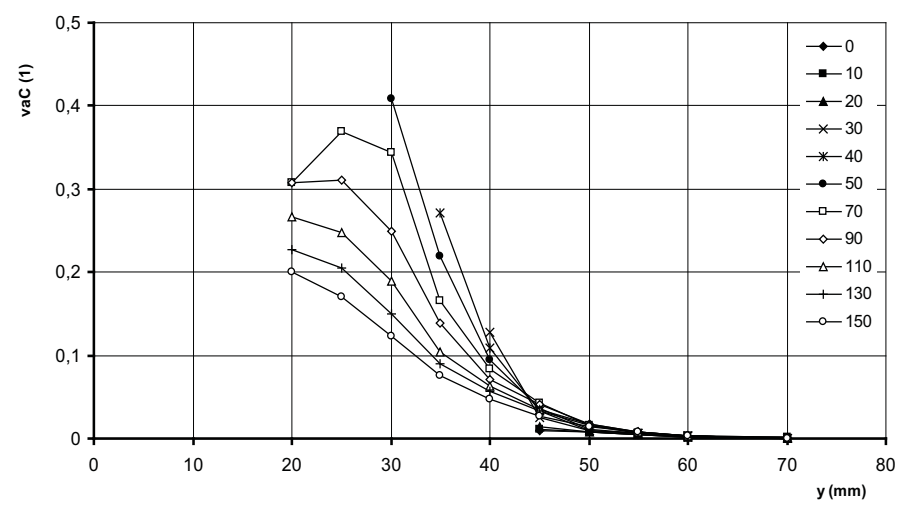

Graph 3: Fluctuations of the concentration $\operatorname{var}(C)(x$-profiles).

Profiles of the molar concentration over the hill were calculated using described procedure. The mean values show Graph 2 and the variances show Graph 3.

\section{Conclusion}

Statistical moments of the velocity and the scalar quantity fluctuations can be obtained from CTA hot-wire measurement. A procedure of simultaneous measurement of the velocity and the scalar quantity with dual hot-wire probe is described. Application of the procedure to a velocity-concentration case employing two parallel wires works satisfactory. Distributions of mean values and variances of the molar concentration over a two dimensional polynomial-shaped hill is shown in the paper.

\section{ACKNOWLEDGEMENT}

This work is supported by GA CR projects GA101/08/1112 and GPP101/10/P556. Support is gratefully acknowledged. 


\section{REFERENCES}

[1] Way J., Libby P.A.: Hot-Wire Probes for Measuring Velocity and Concentration in Helium-Air Mixtures. AIAA Journal, 8, 5, pp.976-978. 1970.

[2] Chassaing P.: Heat transfer from cylindrical anemometer probes in $\mathrm{CO}_{2}$-air mixtures. Physics of Fluids, vol.20, nr 8, pp. 1260-1262. 1977.

[3] Sakai Y., Watanabe T., Kamohara S., Kushida T., Nakanuta I.: Simultaneous measurements of concentration and velocity in a $\mathrm{CO}_{2}$ jet issuing into a grid turbulence by two-sensor hot-wire probe. Int. J. of Heat and Fluid Flow, 22, pp.227-236. 2001.

[4] Jonáš P., Mazur O., Moryń-Kucharczyk E., Podolski M.: The spreading of a carbon dioxide gas round jet into a collateral air flow. Proc. Conference on Modelling Fluid Flow, Budapest, 297-303. 2006.

[5] Almeida G.P., Durao D.F.G, Heitor M.V.: Wake flows behind two dimensional model hills. Exp. Thermal and Fluid Science, 7: 87-101. 1993. 